\title{
A SURVEY OF SPECIAL ARONSZAJN TREES
}

\section{RADEK HONZÍK and ŠÁRKA STEJSKALOVÁ*}

Dept. of Logic, Faculty of Arts, Charles University in Prague

*E-mail: sarka@logici.cz

\begin{abstract}
The paper reviews special Aronszajn trees, both at $\omega_{1}$ and $\kappa^{+}$for an uncountable regular $\kappa$. It provides a comprehensive classification of the trees and discusses the existence of these trees under different set-theoretical assumptions. The paper provides details and proofs for many folklore results which circulate (often without a proper proof) in the literature.
\end{abstract}

Keywords: special Aronszajn trees

\section{Introduction}

A tree, which is now called Aronszajn, was first constructed by Nachman Aronszajn and the construction can be found in [Kur35]. The constructed tree was actually a special $\omega_{1}$-Aronszajn tree. The definition of special Aronszajn tree has several equivalent variants and in the literature can be found many generalizations of the definition of a special Aronszajn tree. In this paper we focus on the question what are the relationships between them and provide a basic classification.

\subsection{Preliminaries}

In this section, we provide a review of basic definitions and facts relating to trees.

Definition 1.1. We say that $(T,<)$ is a tree if $(T,<)$ is a partial order such that for each $t \in T$, the set $\{s \in T \mid s<t\}$ is wellordered by $<$.

Definition 1.2. We say that $S \subseteq T$ is a subtree of $(T,<)$ in the induced ordering $<$ if $\forall s \in S \forall t \in T(t<s \rightarrow t \in S)$.

Definition 1.3. Let $T$ be a tree

(i) If $t \in T$, then $h t(t, T)=o t(\{s \in T \mid s<t\})$ is height of $t$ in $T$;

(ii) For each ordinal $\alpha$, we define the $\alpha$-th level of $T$ as $T_{\alpha}=\{t \in T \mid h t(t)=\alpha\}$;

(iii) The height of $T, \operatorname{ht}(T)$, is the least $\alpha$ such that $T_{\alpha}=\varnothing$;

(iv) $T \uparrow \alpha=\bigcup_{\beta<\alpha} T_{\beta}$ is a subtree of $T$ of height $\alpha$.

Definition 1.4. For a regular $\kappa \geq \omega, T$ is called a $\kappa$-tree if $T$ has height $\kappa$, and $\left|T_{\alpha}\right|<\kappa$ for each $\alpha<\kappa$. 
Many $\kappa$-tree is isomorphic to a subtree of the full tree $\left({ }^{<\kappa} \kappa, \subset\right)$. More precisely, this is the case whenever the $\kappa$-tree is normal. See the definition below.

Definition 1.5. A normal $\kappa$-tree is a tree $T$ such that:

(i) $h t(T)=\kappa$;

(ii) $\left|T_{\alpha}\right|<\kappa$, for every $\alpha<\kappa$;

(iii) $\left|T_{0}\right|=1$;

(iv) If $h t(s, T)=h t(t, T)$ is a limit ordinal, then $s=t$ if and only if $\{r \in T \mid r<s\}=$ $\{r \in T \mid r<t\}$.

Note that the conditions (i) and (ii) ensure that a normal $\kappa$-tree is a $\kappa$-tree.

Fact 1.6. Let $\kappa$ be a regular cardinal. Then every normal $\kappa$-tree is isomorphic to a subtree $T^{\prime}$ of the full tree $\left({ }^{<\kappa} \kappa, \subset\right)$.

If we consider a successor cardinal $\kappa^{+}$in the previous fact, then the levels of the $\kappa^{+}$-tree have size $\leq \kappa$. Hence we can strengthen the formulation of the previous fact for successor cardinals as follows: Every normal $\kappa^{+}$-tree is isomorphic to a subtree $T^{\prime}$ of the full tree $\left(<\kappa^{+} \kappa, \subset\right)$.

Definition 1.7. Let $T$ be a tree. We say that $B$ is a branch if it is a maximal chain in $T$.

Definition 1.8. Let $\kappa$ be a regular cardinal. We say that a $\kappa$-tree $T$ is a $\kappa$-Aronszajn tree if it has no branch of size $\kappa$. We denote the class of all Aronszajn trees at $\kappa$ as $\mathbb{A}(\kappa)$.

By König's Lemma, no $\omega$-Aronszajn trees exist. On the other hand, by result of Aronszajn, there exists an $\omega_{1}$-Aronszajn tree. Moreover, if we assume $\mathrm{GCH}$, then there exists a $\kappa^{+}$-Aronszajn tree for each regular cardinal $\kappa$, by a result of Specker [Spe49].

There are two common strengthenings of the notion of an Aronszajn tree. The first one leads to the notion of a special Aronszajn tree, to which we dedicate the next section. The second leads to the notion of a Suslin tree.

Definition 1.9. Let $\kappa$ be a regular cardinal. We say that a $\kappa$-Aronszajn tree is Suslin, if it has no antichain ${ }^{1}$ of size $\kappa$. We denote the class of all Suslin trees at $\kappa$ as $\mathbb{S}(\kappa)$.

The notion of an $\omega_{1}$-Suslin tree first appeared in connection with the Suslin problem of the characterization of the real line. Actually, in [Kur35] Kurepa showed that the original Suslin hypothesis $(\mathrm{SH})$ can be formulated as the claim that there are no Suslin trees. For more details about Suslin hypothesis see [Jec03].

\section{Special Aronszajn trees at $\omega_{1}$}

\subsection{Classification}

In this section, we classify different types of special Aronszajn trees at $\omega_{1}$. Most of the notions are standard, but dispersed through diverse papers, so we think it is useful to provide a unified treatment here.

$1 A \subset T$ is an antichain if for every $t, s \in A$, if $t \neq s$, then there is no $u \in T$ such that $u \geq t$ and $u \geq s$. 
Definition 2.1. We say that an $\omega_{1}$-Aronszajn tree $T$ is special if $T$ is a union of countable many antichains. We denote the class of all special Aronszajn trees at $\omega_{1}$ as $\mathbb{A}^{s p}\left(\omega_{1}\right)$.

Definition 2.2. Let $\kappa$ be a regular cardinal, $T$ be a $\kappa$-Aronszajn tree and $\mathbb{P}=\left\langle P,\left\langle_{\mathbb{P}}\right\rangle\right.$ be a partially ordered set. We say that $T$ is $\mathbb{P}$-embeddable if there is a function $f: T \rightarrow P$ such that $s<_{T} t \rightarrow f(s)<_{\mathbb{P}} f(t)$. We denote the class of all $\mathbb{P}$-embeddable trees at $\kappa$ as $\mathbb{T}(\mathbb{P})(\kappa)$.

Note that the previous definition can be generalized for arbitrary partially ordered set.

Definition 2.3. Let $\kappa$ be a regular cardinal, $\mathbb{R}=\left\langle R,<_{\mathbb{R}}\right\rangle$ and $\mathbb{P}=\left\langle P,<_{\mathbb{P}}\right\rangle$ be a partially ordered sets. We say that $\mathbb{R}$ is $\mathbb{P}$-embeddable if there is a function $f: R \rightarrow P$ such that $s<_{\mathbb{R}} t \rightarrow f(s)<_{\mathbb{P}} f(t)$.

Fact 2.4. The following are equivalent for an $\omega_{1}$-Aronszajn tree $T$ :

(i) $T$ is special;

(ii) There is $f: T \rightarrow \omega$ such that if $s$, $t$ are comparable in $T$, then $f(s) \neq f(t)$;

(iii) $T$ is $\mathbb{Q}$-embeddable, i.e. $T \in \mathbb{T}(\mathbb{Q})\left(\omega_{1}\right)$.

When we work with $\mathbb{Q}$-embeddable Aronszajn trees it is natural to consider also $\mathbb{R}$ embeddable Aronszajn trees and ask what is the connection between them. The following fact tells us how to characterise $\mathbb{R}$-embeddable Aronszajn trees using $\mathbb{Q}$-embeddable Aronszajn trees. It was first proved in [Bau70].

Fact 2.5. Let $T$ be an $\omega_{1}$-tree. $T$ is $\mathbb{R}$-embeddable if and only if $T^{*}=\bigcup_{\alpha<\omega_{1}} T_{\alpha+1}$ is Q-embeddable.

Now, we introduce the concept of an M-special Aronszajn tree.

Definition 2.6. We say that an $\omega_{1}$-Aronszajn tree $T$ is $\mathrm{M}$-special if $T$ is isomorphic to the subtree of $\left\{s \in{ }^{<\omega_{1}} \omega \mid s\right.$ is 1-1 $\}$. We denote the class of all M-special $\omega_{1}$-Aronszajn trees as $\mathbb{A}^{\mathrm{M}-s p}\left(\omega_{1}\right)$.

We use the notation M-special to distinguish special Aronszajn trees defined by Mitchell in [Mit72] from now more used Definition 2.1. Note that Mitchell's definition includes just normal trees in contrast to Definition 2.1. In this sense the notion of a special tree is more general than M-special. However, if we consider just normal trees, then every special normal tree can be represented by an $\mathrm{M}$-special tree. The converse may not hold in general, see Lemma 2.16 .

Lemma 2.7. If $T$ is a normal special $\omega_{1}$-Aronszajn tree, then $T$ is $\mathrm{M}$-special.

Proof. Fix for every $\alpha<\omega_{1}$ a 1-1 function $g_{\alpha}: T_{\alpha} \rightarrow \omega$, and write $T=\bigcup_{n<\omega} A_{n}$, where $A_{n}$ is an antichain for each $n<\omega$.

We define by induction on $\alpha<\omega_{1}$ a tree $T^{\prime}$ and an isomorphism $i: T \rightarrow T^{\prime}$, where $T^{\prime}$ is a subtree of $\left\{s \in{ }^{<\omega_{1}}(\omega \times \omega) \mid s\right.$ is 1-1 $\}$. The isomorphism $i$ will be a union of partial isomorphisms $i_{\alpha}: T \uparrow \alpha \rightarrow T^{\prime} \uparrow \alpha$.

Set $T_{0}^{\prime}=\{\varnothing\}$ and $i_{1}(r)=\varnothing$, where $r$ is the root of $T$. As we assume that $T$ is normal, $i_{1}$ is an isomorphism between $T \uparrow 1$ and $T^{\prime} \uparrow 1$.

Suppose that we have constructed $i_{\beta}: T \uparrow \beta \rightarrow T^{\prime} \uparrow \beta$ for each $\beta<\alpha$. First, if $\alpha$ is limit, set $i_{\alpha}=\bigcup_{\beta<\alpha} i_{\beta}$ and $T^{\prime} \uparrow \alpha=\bigcup_{\beta<\alpha} T^{\prime} \uparrow \beta$. 
If $\alpha=\gamma+1$ and $\gamma$ is a successor, then we define $i_{\alpha}$ by extending $i_{\gamma}$ setting for each $s \in T_{\gamma}:$

$$
i_{\alpha}(s)=i_{\gamma}(t) \cup\left\{\left\langle\gamma,\left\langle g_{\gamma}(s), n\right\rangle\right\rangle\right\},
$$

where the node $t$ is the immediate predecessor of $s$ and $s \in A_{n}$. Let $T^{\prime}\left\lceil\alpha=T^{\prime} \uparrow\right.$ $\gamma \cup T_{\gamma}^{\prime}$, where $T_{\gamma}^{\prime}=\left\{i_{\alpha}(s) \mid s \in T_{\gamma}\right\}$. It is clear that each function in $T_{\gamma}^{\prime}$ is 1-1 since each two comparable nodes must be in different antichains.

If $\alpha=\gamma+1$ and $\gamma$ is limit, then we define $i_{\alpha}$ by extending $i_{\gamma}$ setting for each $s \in T_{\gamma}$ :

$$
i_{\alpha}(s)=\bigcup\left\{i_{\gamma}(t) \mid t<s\right\} .
$$

By (iv) of Definition 1.5, $i_{\alpha}$ is 1-1 and clearly it is also an isomorphism. Let $T^{\prime} \uparrow \alpha=T^{\prime} \uparrow$ $\gamma \cup T_{\gamma}^{\prime}$, where $T_{\gamma}^{\prime}=\left\{i_{\alpha}(s) \mid s \in T_{\gamma}\right\}$. Again it is obvious that each function in $T_{\gamma}^{\prime}$ is 1-1 since it is a union of 1-1 functions with gradually increasing domains.

At the end, set $T^{\prime}=\bigcup_{\alpha<\omega_{1}} T^{\prime} \uparrow \alpha$ and $i=\bigcup_{\alpha<\omega_{1}} i_{\alpha}$. It is easy to see that the tree $T^{\prime}$ is isomorphic to a subtree of $\left\{s \in{ }^{<\omega_{1}} \omega \mid s\right.$ is 1-1 $\}$ by any bijection between $\omega \times \omega$ and $\omega$. Hence $T$ is M-special.

Note that at limit steps we use just the assumption that the tree is normal. Hence we can generalize this lemma to $\mathbb{R}$-embeddable trees. The proof of the implication from right to left can be found in [Dev72].

Lemma 2.8. Let $T$ be an $\omega_{1}$-Aronszajn tree. $T$ is normal $\mathbb{R}$-embeddable if and only if $T$ is M-special.

Proof. $(\Rightarrow)$ Let $T$ be a normal $\mathbb{R}$-embeddable. Then $T^{*}=\bigcup_{\alpha<\omega_{1}} T_{\alpha+1}$ is $\mathbb{Q}$ embeddable and so $T^{*}=\bigcup_{n<\omega} A_{n}$ where $A_{n}$ is an antichain for each $n$. The rest of the proof is the same as the proof of Lemma 2.7 since we used the antichains only in the successor step.

$(\Leftarrow)$ Let $T$ be $M$-special. We define $f: T \rightarrow \mathbb{R}$ by setting

$$
f(t)=\sum_{i=0}^{\infty} \frac{\mathcal{X}_{\mathrm{Rng}(t)}(i)}{10^{i}},
$$

where $X_{X}$ is the characteristic function of a set $X \subset \omega$. Since every node of $T$ is a 1-1 function from some ordinal $\alpha<\omega_{1}$ to $\omega$, if $s<t$ then $\operatorname{Rng}(s) \subset \operatorname{Rng}(t)$ and so there is $n<\omega$ such that $0=X_{\operatorname{Rng}(s)}(n)<\mathcal{X}_{\mathrm{Rng}(t)}(n)=1$ and $\mathcal{X}_{\mathrm{Rng}(s)}\left\lceil n=\mathcal{X}_{\mathrm{Rng}(t)}\lceil n\right.$. Hence $f(s)<f(t)$.

By Fact 2.5, if the tree $T$ is $\mathbb{R}$-embeddable then $T\left\lceil S\right.$ for $S=\left\{\alpha+1 \mid \alpha<\omega_{1}\right\}$ is $\mathbb{Q}$ embeddable. So it is natural to introduce the concept of $S$-special for arbitrary unbounded subset of $S \subseteq \omega_{1}$. The following definition is from [She98].

Definition 2.9. Let $S$ be an unbounded subset of $\omega_{1}$. We say that an $\omega_{1}$-tree $T$ is $S$-special if $T \uparrow S$ is $\mathbb{Q}$-embeddable, where

$$
T \uparrow S=\{t \in T \mid h t(t, T) \in S\},
$$


with the induced ordering. We say that an $\omega_{1}$-tree $T$ is $\mathcal{S}$-special if there is $S$, an unbounded subset of $\omega_{1}$, such that $T$ is $S$-special. We denote the class of all $\mathcal{S}$-special $\omega_{1}$-Aronszajn trees as $\mathbb{A}^{\mathcal{S} \text {-sp }}\left(\omega_{1}\right)$.

The following fact from [DJ74] says that if we only consider $S$-special trees for closed unbounded subsets $S$ of $\omega_{1}$, we get nothing new.

Fact 2.10. Let $C$ be a closed unbounded subset of $\omega_{1}$. If $T$ is a $C$-special $\omega_{1}$-Aronszajn tree, then $T$ is special.

The following Fact 2.11, which can be found in [She98], says that if all Aronszajn trees are $S$-special for some given unbounded subset of $\omega_{1}$, then all of them are in fact special. As an easy corollary, we have by Fact 2.5 that if every $\omega_{1}$-Aronszajn tree is $\mathbb{R}$-embeddable, then every $\omega_{1}$-Aronszajn tree is $\mathbb{Q}$-embeddable.

Fact 2.11. Let $S$ be an unbounded subset of $\omega_{1}$. If every $\omega_{1}$-Aronszajn tree is $S$-special then every $\omega_{1}$-Aronszajn tree is special. In particular, if every $\omega_{1}$-Aronszajn tree is $\mathbb{R}$ embeddable, then every $\omega_{1}$-Aronszajn tree is $\mathbb{Q}$-embeddable.

Note that $\mathcal{S}$-special Aronszajn trees, including special, $\mathbb{R}$-embeddable, and M-special Aronszajn trees, are not Suslin in the following strong sense: every uncountable subset of such tree contains an uncountable antichain. This motivates the following definition.

Definition 2.12. We say that an $\omega_{1}$-tree $T$ is non-Suslin if every uncountable subset $U$ of $T$ contains an uncountable antichain. We denote the class of all non-Suslin Aronszajn trees at $\omega_{1}$ as $\mathbb{A}^{N S}\left(\omega_{1}\right)$.

The name of non-Suslin trees is inspired by the fact that every non-Suslin tree is not Suslin. On the other hand, every tree that is not non-Suslin has a Suslin subtree, as follows from the next fact that can be found in [Han81].

Fact 2.13. Let $T$ be an $\omega_{1}$-Aronszajn tree. If $T$ is not non-Suslin, then $T$ has a subtree which is Suslin.

Lemma 2.14. Let $T$ be an $\omega_{1}$-Aronszajn tree. If $T$ is $\mathcal{S}$-special, then $T$ is non-Suslin.

Proof. Assume for contradiction that $T$ is an $\mathcal{S}$-special $\omega_{1}$-Aronszajn tree which is not non-Suslin. By the previous fact $T$ has a subtree $T^{\prime}$ which is Suslin. Since $T$ is $\mathcal{S}$-special, $T^{\prime}$ is $\mathcal{S}$-special, too. Hence there is an unbounded subset $S$ of $\omega_{1}$ such that $T^{\prime}\lceil S=$ $\bigcup_{n<\omega} A_{n}$, where $A_{n}$ is an antichain for each $n$. By pigeon-hole principle, for some $n<\omega$ the size of $A_{n}$ must be greater than $\omega$. This contradicts the fact that $T^{\prime}$ is Suslin.

To sum up, for general trees we obtain:

$$
\mathbb{A}^{s p}\left(\omega_{1}\right)=\mathbb{T}(\mathbb{Q})\left(\omega_{1}\right) \subseteq \mathbb{T}(\mathbb{R})\left(\omega_{1}\right) \subseteq \mathbb{A}^{\mathcal{S}-s p}\left(\omega_{1}\right) \subseteq \mathbb{A}^{N S}\left(\omega_{1}\right) .
$$

If we consider only normal trees, we get:

$$
\mathbb{A}^{s p}\left(\omega_{1}\right)=\mathbb{T}(\mathbb{Q})\left(\omega_{1}\right) \subseteq \mathbb{T}(\mathbb{R})\left(\omega_{1}\right)=\mathbb{A}^{\mathrm{M}-s p}\left(\omega_{1}\right) \subseteq \mathbb{A}^{\mathcal{S}-s p}\left(\omega_{1}\right) \subseteq \mathbb{A}^{N S}\left(\omega_{1}\right) .
$$

In the next section, for each of these inclusions, we examine if there is a model in which it is proper. 
The existence of special Aronszajn trees at $\omega_{1}$ can be proved in ZFC and by Baumgartner's theorem published in [BMR70] it is consistent with ZFC that every Aronszajn tree at $\omega_{1}$ is special, so $\mathbb{A}^{s p}\left(\omega_{1}\right)=\mathbb{T}(\mathbb{R})\left(\omega_{1}\right)=\mathbb{A}^{\mathcal{S}-s p}\left(\omega_{1}\right)=\mathbb{A}^{N S}\left(\omega_{1}\right)$ is consistent with ZFC. On the other hand, consistently, each inclusion can be proper.

The following Fact 2.15 was first published in [Bau70]. It says that it is consistent that there is an Aronszajn tree which is M-special but not special. As a corollary we obtain that the first inclusion in (2.3) can be consistently proper.

Fact 2.15. Assume $\diamond$. Then there is a non-special Aronszajn tree which is a subtree of $\left\{s \in{ }^{<\omega_{1}} \omega \mid s\right.$ is $\left.1-1\right\}$. In particular, there is an $\mathbb{R}$-embeddable $\omega_{1}$-Aronszajn tree which is not special.

Proof. This has been proved by Baumgartner (see [Dev72]). We have extended his proof to obtain a more general result, see Theorem 3.27.

The following lemma is a consequence of Fact 2.15 and it shows us that the second inclusion in (2.3) can be consistently proper.

Lemma 2.16. Assume $\diamond$. Then there is an $\omega_{1}$-Aronszajn tree, which is $\mathcal{S}$-special and not $\mathbb{R}$-embeddable.

Proof. By Fact 2.15, assuming $\diamond$, there is an $\omega_{1}$-Aronszajn tree which is $\mathbb{R}$-embeddable, but not $\mathbb{Q}$-embeddable. Let $\alpha<\omega_{1}$ be a limit ordinal and let $t \in T_{\alpha}$. For the chain $C=$ $\{s \in T \mid s<t\}$ we add a new node $t_{C}$ such that $t_{C}<t$ and $t_{C}>s$ for all $s \in C$. Consider the tree $T^{\prime}$ which is created by adding such $t_{C}$ for every limit node $t$. Note that $\bigcup_{\alpha<\omega_{1}} T_{\alpha+1}^{\prime}=$ $T \backslash T_{0}$. Now, $T^{\prime}$ is not $\mathbb{R}$-embeddable since $\bigcup_{\alpha<\omega_{1}} T_{\alpha+1}^{\prime}$ is not $\mathbb{Q}$-embeddable. But $T^{\prime}$ is $S$-special for $S=\left\{\alpha+2 \mid \alpha<\omega_{1}\right\}$ since $T^{\prime} \uparrow S=\bigcup_{\alpha<\omega_{1}} T_{\alpha+1} \backslash T_{1}$.

The claim that the last inclusion in (2.3) can be consistently proper is a consequence of the theorem published in [Sch14], which says that if ZFC is consistent, so is $\mathrm{ZFC}+\mathrm{SH}^{2}$ + there is an Aronszajn tree $T$ at $\omega_{1}$ which is not $\mathcal{S}$-special. If $\mathrm{SH}$ holds, then by Fact 2.13 every Aronszajn tree is non-Suslin. Therefore $T$ is non-Suslin and it witnesses that ZFC $+\mathbb{A}^{\mathcal{S}-s p}\left(\omega_{1}\right) \neq \mathbb{A}^{N S}\left(\omega_{1}\right)$ is consistent.

\section{Special Aronszajn trees at larger $\kappa$}

\subsection{Generalisations of $\mathbb{Q}$}

In this section we consider some common generalisations of $\mathbb{Q}$ at higher cardinals. The following definitions of $\mathbb{Q}_{\kappa}$ and $\mathbb{Q}_{\kappa}^{*}$ are taken from [Tod84]. In addition, we introduce our definition of a generalisation of the real line for higher cardinals because we want to generalize the concept of an $\mathbb{R}$-embeddable tree (see Definition 2.2).

2 Suslin Hypothesis 
Definition 3.1. Let $\kappa$ be a regular cardinal. Then

$$
\begin{aligned}
& \mathbb{Q}_{\kappa}^{*}=\left(\left\{f \in{ }^{\omega} \mathcal{K} \mid\{n<\omega \mid f(n) \neq 0\} \text { is finite }\right\} \backslash\{\overline{0}\},<_{\text {lex }}\right) ; \\
& \mathbb{Q}_{\kappa}=\left(\left\{f \in{ }^{\kappa} 2||\{\alpha<\kappa \mid f(\alpha) \neq 0\} \mid<\kappa\right\} \backslash\{\overline{0}\},<_{\text {lex }}\right) ; \\
& \mathbb{R}_{\kappa}=\left(\left\{f \in{ }^{\kappa} 2 \mid(\neg \exists \alpha<\kappa)[f(\alpha)=0 \text { and }(\forall \beta>\alpha)(f(\beta)=1)]\right\} \backslash\{\overline{0}, \overline{1}\},<_{\text {lex }}\right) ;
\end{aligned}
$$

where $<_{\text {lex }}$ is the lexicographical ordering, $\overline{0}(\overline{1})$ denotes the sequence of zeros (ones) of length $\omega$ in (3.1) and of length $\kappa$ in (3.2) and (3.3).

Note that in the definition of $\mathbb{R}_{\kappa}$, we allow all 1's on a tail, but restrict this configuration by demanding that in this case there is no greatest $\alpha$ with $f(\alpha)=0 .{ }^{3}$

Remark 3.2. Note that $\mathbb{Q}_{\omega} \cong \mathbb{Q} \cong \mathbb{Q}_{\omega}^{*}$. On the other hand, for $\kappa>\omega, \mathbb{Q}_{\kappa} \neq \mathbb{Q}_{\kappa}^{*}$, even if $\left|\mathbb{Q}_{\kappa}\right|=\kappa$. This holds, because $\mathbb{Q}_{\kappa}^{*}$ does not contain any decreasing sequence of uncountable length. However, in $\mathbb{Q}_{\kappa}$ there are decreasing sequences of length $\kappa$.

In this paper we work mainly with $\mathbb{Q}_{\kappa}$ because it has some nice properties: in particular, one can generalize Kurepa's Theorem for $\mathbb{Q}_{\kappa}$ and prove Lemma 3.4 which is very useful and plays the key role in proving Lemma 3.13. On the other hand, the main advantage of $\mathbb{Q}_{\kappa}^{*}$ is that it always has size $\kappa$. When we work with $\mathbb{Q}_{\kappa}$, we need to assume that $\kappa^{<\kappa}=\kappa$ to control its size.

The following easy lemma tells us that $\mathbb{Q}_{\kappa}$ has the properties which we want from a generalisation of $\mathbb{Q}$, with the exception that it does not have to have size $\kappa$. The proof is left as an exercise.

Lemma 3.3. The ordering $\mathbb{Q}_{\kappa}$ is linear, dense, without endpoints and $\left|\mathbb{Q}_{\kappa}\right|=\kappa^{<\kappa}$.

There is an asymmetry in $\mathbb{Q}_{\kappa}$ between decreasing and increasing sequences:

Lemma 3.4. Assume $\kappa>\omega$ is regular.

(i) Let $A=\left\langle f_{\alpha} \mid \alpha<\lambda\right\rangle$ be a strictly decreasing sequence in $\mathbb{Q}_{\kappa}$, where $\lambda$ is a limit ordinal such that $\omega \leq \lambda<\kappa$. Then $A$ does not have an infimum in $\mathbb{Q}_{\kappa}$.

(ii) Let $B=\left\langle g_{\alpha} \mid \alpha<\lambda\right\rangle$ be a strictly increasing sequence in $\mathbb{Q}_{\kappa}$ where $\lambda$ is a limit ordinal such that $\omega \leq \lambda<\kappa$. Then $B$ has a supremum in $\mathbb{Q}_{\kappa}$.

Proof. Ad (i). Let $A=\left\langle f_{\alpha} \mid \alpha<\lambda\right\rangle$ be given. Assume for contradiction that there is the infimum $f \in \mathbb{Q}_{\kappa}$ of $A$. Since $f \in \mathbb{Q}_{\kappa}$, there is $\beta_{0}<\kappa$ such that for each $\beta \geq \beta_{0} f(\beta)=0$. Since $\lambda<\kappa$ and $\kappa$ is regular, there is $\gamma_{0}<\kappa$ such that for each $\gamma \geq \gamma_{0}$ and for each $\alpha<\lambda$ $f_{\alpha}(\gamma)=0$. Let $\delta=\max \left\{\beta_{0}, \gamma_{0}\right\}$. We define $f^{*}=f\lceil\delta \cup\{\langle\delta, 1\rangle\} \cup\{\langle\beta, 0\rangle \mid \beta>\delta\}$. Clearly, $f^{*}>f$. Since $f<f_{\alpha}$ for every $\alpha<\lambda$ and since $\delta=\max \left\{\beta_{0}, \gamma_{0}\right\}, f^{*}<f_{\alpha}$ for every $\alpha<\lambda$. This is a contradiction because we assume that $f$ is the infimum of $A$.

Ad (ii). Let $B=\left\langle g_{\alpha} \mid \alpha<\lambda\right\rangle$ be given. We define supremum $g$ by induction on $\beta<\kappa$.

For $\beta=0$. Set

$$
g(0)= \begin{cases}1 & \text { if } \exists \alpha<\lambda\left(g_{\alpha}(0)=1\right) \\ 0 & \text { otherwise. }\end{cases}
$$

3 If $f \in{ }^{\kappa} 2$ does not satisfy (3.3) and $\alpha$ is the greatest position with 0 , then we can define $g \in \mathbb{R}_{\kappa}$ which is the immediate successor of $f$ in the lexicographical order: define $g$ exactly as $f$ below $\alpha$, and set $g(\beta)=1$ for all $\beta \geq \alpha$. To prohibit this situation (which violates density of the ordering), we choose to disallow such $f$ 's in (3.3). If there is no greatest $\alpha$ where $f(\alpha)=0$, this problem does not arise. 
Assume that $g\lceil\beta$ is defined, then we define $g(\beta)$ as follows:

$$
g(\beta)= \begin{cases}1 & \text { if } \exists \alpha<\lambda \text { such that } g_{\alpha}(\beta)=1 \text { and } g_{\alpha}\lceil(\beta+1)>g\lceil\beta \cup\{\langle\beta, 0\rangle\} ; \\ 0 & \text { otherwise. }\end{cases}
$$

First note that $g$ is in $\mathbb{Q}_{\kappa}$ since $\kappa$ is regular and $\lambda<\kappa$.

Now, we show that $g$ is the supremum of $B$. It is obvious that $g_{\alpha}<g$ for every $\alpha<\lambda$. Hence it is enough to show that $g$ is the least upper bound of $B$. Let $h<g$ be given. Then there is $\beta_{0}<\kappa$ such that $h\left\lceil\beta_{0}=g\left\lceil\beta_{0}\right.\right.$ and $0=h\left(\beta_{0}\right)<g\left(\beta_{0}\right)=1$. By definition of $g$ there is $\alpha$ such that $g_{\alpha}\left\lceil\left(\beta_{0}+1\right)>g\left\lceil\beta_{0} \cup\left\langle\beta_{0}, 0\right\rangle\right.\right.$. As $h\left\lceil\beta_{0}=g\left\lceil\beta_{0}\right.\right.$ and $h\left(\beta_{0}\right)=0$, $g\left\lceil\beta_{0} \cup\left\langle\beta_{0}, 0\right\rangle=h \uparrow\left(\beta_{0}+1\right)\right.$ and so $g_{\alpha} \uparrow\left(\beta_{0}+1\right)>h \uparrow\left(\beta_{0}+1\right)$. Therefore $g_{\alpha}>h$.

Note that it was important in (i) of the previous lemma that $\lambda$ is a limit ordinal $<\kappa$. One can easily find decreasing sequences in $\mathbb{Q}_{\kappa}$ of length $\kappa$ which do have the infimum. ${ }^{4}$

Now, we present the generalisation of Kurepa's Theorem for $\mathbb{Q}_{K}$ :

Theorem 3.5. (Generalised Kurepa's Theorem) Assume $\kappa^{<\kappa}=\kappa$. Let $(E,<)$ be a partially ordered set. Then the following are equivalent:

(i) $E$ is embeddable in $\mathbb{Q}_{\kappa}$;

(ii) $E$ is the union of at most $\kappa$-many antichains.

Proof. (i) $\Rightarrow$ (ii) Let $f$ be the embedding. Let $\left\{q_{\alpha} \mid \alpha<\kappa\right\}$ be an enumeration of $\mathbb{Q}_{\kappa}$. We define $A_{\alpha}=f^{-1}\left(q_{\alpha}\right)$ for each $q_{\alpha} \in \operatorname{Rng}(f)$. Obviously, each $A_{\alpha}$ is an antichain since $f$ is an embedding.

(ii) $\Rightarrow$ (i) We assume that $\bigcup_{\alpha<\kappa} A_{\alpha}=E$, where each $A_{\alpha}$ is an antichain. Moreover, without loss of generality, we may assume that for each $\beta, \alpha<\kappa, A_{\alpha} \cap A_{\beta}=\varnothing$. Let $f: E \rightarrow \kappa$ be a function such that $A_{\alpha}=f^{-1}(\alpha)$. For $x \in E$ define $g(x)$ so that $g(x)(\alpha)=1$ if and only if $\alpha \leq f(x)$ and $\{y \in E \mid y \leq x\} \cap A_{\alpha} \neq \varnothing$.

Notice that $g(x)$ is in $\mathbb{Q}_{\kappa}$ because $g(x)(\alpha)=1$ implies that $\alpha \leq f(x)$, where $f(x) \in \kappa$.

Now, we check that $g$ is an embedding. Assume that $x<y$ are in $E$ and $x \in A_{\alpha}, y \in A_{\beta}$ for some $\beta \neq \alpha$. We distinguish two cases.

First suppose that $\alpha<\beta$. Then $g(x)(\alpha)=1$ and also $g(y)(\alpha)=1$ since $x<y$ and $x \in A_{\alpha}$. And for all $\gamma<\alpha$ if $g(x)(\gamma)=1$ then $g(y)(\gamma)=1$ and so $g(x)\left\lceil\alpha \leq_{l e x} g(y)\lceil\alpha\right.$. If $g(x)\left\lceil\alpha<_{\text {lex }} g(y) \uparrow \alpha\right.$, then $g(x)<g(y)$ and we are finished. If $g(x)\lceil\alpha=g(y) \uparrow \alpha$, then we can continue as follows: for all $\gamma>\alpha$ it holds that $g(x)(\gamma)=0$ since $\gamma>f(x)$. Hence $g(x)(\beta)=0$ and $g(y)(\beta)=1$; therefore $g(x)<g(y)$.

Next suppose that $\beta<\alpha$. Again for all $\gamma<\beta$, if $g(x)(\gamma)=1$ then $g(y)(\gamma)=1$ and so $g(x)\left\lceil\beta \leq_{\text {lex }} g(y) \mid \beta\right.$. Now, we show that $g(x)(\beta)=0$ and $g(y)(\beta)=1$. Assume for contradiction that $g(x)(\beta)=1$. Then by definition of the function $g$, we know there exists $z \in A_{\beta}$ and $z \leq x$. Hence $z<y$ and this is a contradiction since there are two comparable elements in $A_{\beta}$. By the definition of $g, g(y)(\beta)=1$ and so $g(x)<g(y)$.

Remark 3.6. Note that the assumption $\kappa^{<\kappa}=\kappa$ is necessary just in the proof of (i) $\Rightarrow$ (ii).

4 Compare with $\mathbb{Q}$ : some infinite decreasing sequences have an infimum; since there is no limit ordinal below $\omega$, the analogue of (i) of the previous Lemma does not appear in $\mathbb{Q}$. 
Remark 3.7. We cannot prove Kurepa's Theorem for $\mathbb{Q}_{\kappa}^{*}$, for $\kappa>\omega$ a regular cardinal, since it does not contain strictly decreasing sequence of uncountable length. Consider the ordinal $\kappa$ with reverse ordering $<^{*}$, i.e. $\alpha<^{*} \beta$ that $\alpha<^{*} \beta$ if and only if $\beta \in \alpha$ for $\alpha$, $\beta \in \kappa$. Then $\kappa$ is a union of $\kappa$-many antichains and cannot be embedded to $\mathbb{Q}_{\kappa}^{*}$.

Partials orders from Theorem 3.5 have another useful characterisation. The proof of the following lemma is easy and it is left as an exercise for the reader.

Lemma 3.8. Let $\kappa$ be regular and let $(E,<)$ be a partially ordered set. Then the following are equivalent:

(i) $E$ is the union of at most $\kappa$-many antichains;

(ii) there is $f: E \rightarrow \kappa$ such that if $s$, are comparable in $E$, then $f(s) \neq f(t)$.

Now, we focus on the partial order $\mathbb{R}_{\kappa}$. We show that it has similar properties as $\mathbb{R}$.

Lemma 3.9. The partial order $\mathbb{R}_{\kappa}$ is

(i) linear, without endpoints;

(ii) $\mathbb{Q}_{\kappa}$ is dense in $\mathbb{R}_{\kappa}$;

(iii) Dedekind complete.

Proof. It is easy to verify that $\mathbb{R}_{\kappa}$ satisfies (i).

Ad (ii). Let $f<\mathbb{R}_{\kappa} g$ in $\mathbb{R}_{\kappa}$ be given. Let $\alpha_{0}$ be the least ordinal such that $0=f\left(\alpha_{0}\right)<$ $g\left(\alpha_{0}\right)=1$. By definition of $\mathbb{R}_{\kappa}$, there is the least $\beta_{0}>\alpha_{0}$ such that $f\left(\beta_{0}\right)=0$. Let $h=f\left\lceil\beta_{0} \cup\left\{\left\langle\beta_{0}, 1\right\rangle\right\} \cup\left\{\langle\gamma, 0\rangle \mid \gamma>\beta_{0}\right\}\right.$. It is easy to see that $h \in \mathbb{Q}_{\kappa}$ and $f<\mathbb{R}_{\kappa} h<_{\mathbb{R}_{\kappa}} g$.

$\mathrm{Ad}$ (iii). It is enough to show that every increasing sequence with upper bound has the supremum. First note that each increasing sequence in $\mathbb{R}_{\kappa}$ has cardinality at most $\kappa^{<\kappa}$ since $\mathbb{Q}_{\kappa}$ is dense in $\mathbb{R}_{\kappa}$ as we proved in the previous paragraph. Let $A=\left\langle f_{\alpha} \in \mathbb{R}_{\kappa} \mid \alpha<\lambda\right\rangle$ for some ordinal $\lambda \leq \kappa^{<\kappa}$ be given and let $f \in \mathbb{R}_{\kappa}$ be the upper bound of $A$. Let $F_{C}$ be a choice function from $\mathcal{P}\left(\mathbb{Q}_{\kappa}\right)$ to $\mathbb{Q}_{\kappa}$. We define the sequence $A_{\mathbb{Q}}$ in $\mathbb{Q}_{\kappa}$ as follows:

$$
\left.A_{\mathbb{Q}}=\left\langle g_{\alpha} \in \mathbb{Q}_{\kappa}\right| g_{\alpha}=F_{C}\left(\left\{q \in \mathbb{Q}_{\kappa} \mid f_{\alpha}<q<f_{\alpha+1}\right\}\right) \text { and } \alpha<\lambda\right\rangle .
$$

We show that $A_{\mathbb{Q}_{\kappa}}$ has the supremum $g$ in $\mathbb{R}$ and that $g$ is also the supremum of $A$ in $\mathbb{R}_{\kappa}$. We define a function $g^{*}: \kappa \rightarrow 2$ by induction on $\beta<\kappa$.

For $\beta=0$. Set

$$
g^{*}(0)= \begin{cases}1 & \text { if } \exists \alpha<\lambda\left(g_{\alpha}(0)=1\right) \\ 0 & \text { otherwise }\end{cases}
$$

Let $g^{*} \uparrow \beta$ be defined, then we define $g^{*}(\beta)$ as follows:

$$
g^{*}(\beta)= \begin{cases}1 & \text { if } \exists \alpha<\lambda \text { such that } g_{\alpha}(\beta)=1 \text { and } g_{\alpha}\left\lceil(\beta+1)>g^{*}\lceil\beta \cup\{\langle\beta, 0\rangle\} ;\right. \\ 0 & \text { otherwise. }\end{cases}
$$

Note that $g^{*}$ may not be in $\mathbb{R}_{\kappa}$, but it holds that $g^{*} \neq\{\langle\alpha, 1\rangle \mid \alpha<\kappa\}$ since the sequence has an upper bound in $\mathbb{R}_{\kappa}$.

Now, we need to show that $g^{*}$ is the supremum of $A_{\mathbb{Q}}$ in $\left(2^{\kappa},<_{\text {lex }}\right)$. However, the proof of this is the same as the proof of Lemma 3.4 (ii). Note that in the Lemma 3.4 (ii) 
we used the assumption that the sequence has length less than $\kappa$ just for showing that the supremum is in $\mathbb{Q}_{\kappa}$.

As we mentioned earlier, $g^{*}$ may not be in $\mathbb{R}_{\kappa}$, but note that $g^{*} \neq\{\langle\alpha, 1\rangle \mid \alpha<\kappa\}$. If $g^{*}$ is not in $\mathbb{R}_{\kappa}$, there is $\beta_{0}<\kappa$ such that $g^{*}\left(\beta_{0}\right)=0$ and $g^{*}(\beta)=1$ for every $\beta>\beta_{0}$. Let $\bar{g}=g^{*}\left\lceil\beta_{0} \cup\left\{\left\langle\beta_{0}, 1\right\rangle\right\} \cup\left\{\langle\beta, 0\rangle \mid \beta>\beta_{0}\right\}\right.$. Clearly $\bar{g} \in \mathbb{R}_{\kappa}$ and there is no function between $g^{*}$ and $\bar{g}$ in $2^{\kappa}$. Now we define $g \in \mathbb{R}_{\kappa}$ by

$$
g= \begin{cases}g^{*} & \text { if } g^{*} \in \mathbb{R}_{\kappa} ; \\ \bar{g} & \text { otherwise. }\end{cases}
$$

It is obvious that $g \in \mathbb{R}_{\kappa}$ and since $g^{*}$ is the supremum of $A_{\mathbb{Q}_{\kappa}}$ in $2^{\kappa}, g$ is the supremum of $A_{\mathbb{Q}_{\kappa}}$ in $\mathbb{R}_{\kappa}$.

To finish the proof of the theorem, it suffices to show that $g$ is also the supremum of $A$. The function $g$ is clearly the upper bound of $A$. Now, we show that $g$ is the least upper bound. Let $h<g$. Since $g$ is the supremum of $A_{\mathbb{Q}_{\kappa}}$, there is $q \in A_{\mathbb{Q}_{\kappa}}$, such that $h<q$. But $q<r$ for some $r \in A$ by the definition of $A_{\mathbb{Q}_{\kappa}}$. Hence $h<r$.

\subsection{Classification}

In the previous section we have built the foundations for the investigation of special $\kappa^{+}$-Aronszajn trees for any regular $\kappa$. We introduced the concept of special, $\mathbb{R}$ embeddable, $M$-special and $\mathcal{S}$-special $\omega_{1}$-Aronszajn trees. Now, we generalize these concepts to higher Aronszajn trees, which are in the center of our interest. When we talk about an Aronszajn tree in this section, we mean a $\kappa^{+}$-Aronszajn tree for some regular cardinal $\kappa>\omega$.

Definition 3.10. Let $\kappa$ be a cardinal. We say that $\kappa^{+}$-Aronszajn tree $T$ is special if $T$ is a union of $\kappa$-many antichains. We denote the class of all special Aronszajn trees at $\kappa^{+}$as $\mathbb{A}^{s p}\left(\kappa^{+}\right)$.

As in the previous section, the concept of a special Aronszajn tree has more equivalent definitions. However, we need to be careful when we talk about $\mathbb{Q}_{\kappa}$-embeddability, since this partial order in general does not have to have size $\kappa$.

Lemma 3.11. Let $\kappa$ be regular. The following are equivalent for a $\kappa^{+}$-Aronszajn tree $T$ :

(i) $T$ is special;

(ii) There is $f: T \rightarrow \kappa$ such that if $s$, $t$ are comparable in $T$, then $f(s) \neq f(t)$.

Proof. This is a direct consequence of Lemma 3.8 .

Lemma 3.12. Assume $\kappa^{<\kappa}=\kappa$. Then $\kappa^{+}$-Aronszajn tree $T$ is special if and only if $T$ is $\mathbb{Q}_{\kappa}$-embeddable.

Proof. It follows from Theorem 3.5.

Again as in the previous section, we can characterise $\mathbb{R}_{\mathcal{K}}$-embeddable Aronszajn trees using $\mathbb{Q}_{\mathcal{K}}$-embeddable Aronszajn trees. This is our generalisation of Fact 2.5.

Theorem 3.13. Assume $\kappa^{<\kappa}=\kappa$. Let $T$ be an $\kappa^{+}$-tree. $T$ is $\mathbb{R}_{\kappa}$-embeddable if and only if $T^{*}=\bigcup_{\alpha<\kappa^{+}} T_{\alpha+1}$ is $\mathbb{Q}_{\kappa}$-embeddable. 
Proof. $(\Rightarrow)$ Let $T$ be $\mathbb{R}_{\kappa}$-embeddable and $T^{*}=\bigcup_{\alpha<\kappa^{+}} T_{\alpha+1}$. Let $f$ be the embedding, $t \in T^{*}$ and let $s \in T$ be the immediate predecessor of $t$. We define $f^{\prime}: T^{*} \rightarrow \mathbb{Q}_{\kappa}$ as follows: $f^{\prime}(t)=q$ where $q \in \mathbb{Q}_{\kappa}$ such that $f(s)<q<f(t)$.

$(\Leftarrow)$ Let $T^{*}=\bigcup_{\alpha<\kappa^{+}} T_{\alpha+1}$ be $\mathbb{Q}_{\kappa}$-embeddable and let $f$ be the embedding.

We first define a function $g: \mathbb{Q}_{\kappa} \rightarrow \mathbb{Q}_{\kappa} \times \mathbb{Q}_{\kappa}$ which will "replace" every $q \in \mathbb{Q}_{\kappa}$ with an open interval $\left(g(q)^{1}, g(q)^{2}\right),{ }^{5}$ while preserving the ordering. More precisely, we will define $g$ by induction on $\kappa$ and ensure it satisfies the following for all $q<q^{\prime}$ in $\mathbb{Q}_{\kappa}$ :

$$
g(q)^{2}<g\left(q^{\prime}\right)^{1} .
$$

Enumerate $\mathbb{Q}_{\kappa}$ as $\left\{q_{\beta} \mid \beta<\kappa\right\}$. We will construct by induction on $\alpha<\kappa$ embeddings $g_{\alpha}:\left\{q_{\beta} \mid \beta<\alpha\right\} \rightarrow \mathbb{Q}_{\kappa} \times \mathbb{Q}_{\kappa}$ which will be used to define the final function $g$.

As we will see below, at the successor step, we define $g_{\alpha+1}$ as an extension of $g_{\alpha}$ to $q_{\alpha}$. Suppose $g_{\alpha+1}\left(q_{\alpha}\right)=\left\langle q, q^{\prime}\right\rangle$ for some $q<q^{\prime}$ in $\mathbb{Q}_{\kappa}$. In addition to choosing $q, q^{\prime}$, fix also two elements $a\left(q_{\alpha}\right)<b\left(q_{\alpha}\right)$ in the interval $\left(q, q^{\prime}\right)$ and two sequences as follows: a strictly increasing sequence of elements in $\left(q, q^{\prime}\right)$ of length $\kappa$ converging to $a\left(q_{\alpha}\right)$ and a strictly decreasing sequence of elements in $\left(q, q^{\prime}\right)$ of length $\kappa$ converging to $b\left(q_{\alpha}\right)$. We denote these sequences $\left\langle a\left(q_{\alpha}\right)_{i} \mid i<\kappa\right\rangle$ and $\left\langle b\left(q_{\alpha}\right)_{i} \mid i<\kappa\right\rangle$, respectively.

Now we provide an inductive definition of the functions $g_{\alpha}, \alpha<\kappa$ :

Set $g_{0}=\varnothing$.

Let $\alpha$ be a limit ordinal. Define

$$
g_{\alpha}=\left\{\left\langle q_{\beta},\left\langle a\left(q_{\beta}\right)_{\alpha+1}, b\left(q_{\beta}\right)_{\alpha+1}\right\rangle\right\rangle \mid \beta<\alpha\right\} .
$$

The idea behind this definition is to take the intervals defined in the previous stages of the construction and "shrink" them to get more space. The shrinking of the intervals makes sure that the construction can continue on the successor steps.

At $\alpha+1$, define $g_{\alpha+1}$ by

$$
g_{\alpha+1}=g_{\alpha} \cup\left\{\left\langle q_{\alpha},\left\langle q, q^{\prime}\right\rangle\right\rangle\right\},
$$

for some suitable interval $\left(q, q^{\prime}\right)$, i.e. for all $s<s^{\prime}$ in the domain of $g_{\alpha+1}$, we should have $g_{\alpha+1}(s)^{2}<g_{\alpha+1}\left(s^{\prime}\right)^{1}{ }^{6}$

When all functions $g_{\alpha}, \alpha<\kappa$, have been constructed, set

$$
g=\left\{\left\langle q_{\alpha},\left\langle a\left(q_{\alpha}\right), b\left(q_{\alpha}\right)\right\rangle\right\rangle \mid \alpha<\kappa\right\} .
$$

By the construction, it follows that $g$ is as required.

Now we can finish the proof of the theorem. Define a function $i: \mathbb{Q}_{\kappa} \rightarrow \mathbb{Q}_{\kappa}$ by $i(q)=r$, where $r$ is some element of the open interval $\left(g(q)^{1}, g(q)^{2}\right)$. We define an embedding $f^{\prime}: T \rightarrow \mathbb{R}_{\kappa}$ as follows:

$$
f^{\prime}(t)= \begin{cases}i(f(t)) & \text { if } t \in T_{\alpha+1} \text { for } \alpha<\kappa^{+} \\ \sup \left\{i(f(s)) \mid s<t \text { and } s \in T_{\beta+1} \text { and } \beta<\alpha\right\} & \text { otherwise. }\end{cases}
$$

$5 g(q)^{1}$ denotes the left coordinate and $g(q)^{2}$ the right coordinate of the pair $g(g)$.

6 When defining $g_{\alpha+1}$, we need to ensure that we can map $q_{\alpha}$ into an interval which is disjoint from the intervals $g_{\alpha}(\beta), \beta<\alpha$, while respecting the ordering. Without the shrinking at the limit stages of the construction, the intervals might converge in a way which prevents the definition of $g_{\alpha+1}\left(q_{\alpha}\right)$. 
Now we need to check that the function $f^{\prime}$ is the embedding of $T$ to $\mathbb{R}$. If $s<t$ and $s$, $t \in T^{*}$, then it is easy to see that $f^{\prime}(s)<f^{\prime}(t)$ because $i$ is order-preserving. If $t \in T_{\alpha}$ for $\alpha$ limit, then $f^{\prime}(s)<f^{\prime}(t)$ since $f^{\prime}(t)$ is the supremum. The only interesting case is if $s \in T_{\alpha}$ for $\alpha$ limit and $t \in T_{\alpha+1}$. Then we need to show

$$
f^{\prime}(t)=i(f(t))>\sup \left\{i(f(r)) \mid r<s \text { and } r \in T_{\beta+1} \text { and } \beta<\alpha\right\}=f^{\prime}(s) .
$$

This follows from the construction of $g$. For every $r<s$ it holds that $i(f(r))<q<i(f(t))$ where $q=g(f(t))^{1}$. Hence

$$
f^{\prime}(s)=\sup \left\{i(f(r)) \mid r<s \text { and } r \in T_{\beta+1} \text { and } \beta<\alpha\right\} \leq q<i(f(t))=f^{\prime}(t) .
$$

Definition 3.14. Let $\kappa$ be a cardinal. We say that $\kappa^{+}$-Aronszajn tree $T$ is $\mathrm{M}$-special if $T$ is isomorphic to a subtree of $\left\{s \in{ }^{<\mathcal{K}^{+}} \kappa \mid s\right.$ is $\left.1-1\right\}$

The following lemma is a generalisation of Lemma 2.7, hence we left the proof as an exercise.

Lemma 3.15. Let $\kappa$ be a regular cardinal. If $T$ is a normal special $\kappa^{+}$-Aronszajn tree then $T$ is M-special.

As in the case for $\omega_{1}$, at the limit step we use just the assumption that the tree is normal. Hence we can generalize this lemma to the following lemma. Note that for this we do not need the assumption $\kappa^{<\kappa}=\kappa$ since we use that the tree $\bigcup_{\alpha<\kappa^{+}} T_{\alpha+1}$ is special instead of $\mathbb{Q}_{\kappa}$-embeddable. We explicitly state this lemma here so it is clear that M-special trees are exactly those trees that are normal and whose successor levels form a special tree, as was the case at $\omega_{1}$.

Lemma 3.16. Let $\kappa$ be a regular cardinal. Let $T$ be a normal $\kappa^{+}$-Aronszajn tree. Then $T^{*}=\bigcup_{\alpha<\kappa^{+}} T_{\alpha+1}$ is special if and only if $T$ is M-special.

Proof. $(\Rightarrow)$ Let $T^{*}=\bigcup_{\alpha<\kappa^{+}} T_{\alpha+1}$ be special. Then $T^{*}=\bigcup_{\xi<\kappa} A_{\xi}$ where $A_{\xi}$ is an antichain for each $\xi<\kappa$. The rest of the proof is the same as the proof of Lemma 3.15.

$(\Leftarrow)$ Let $T$ be an M-special tree. Then $T$ is isomorphic to a subtree $T^{\prime}$ of $\left\{s \in<^{+} \kappa \mid s\right.$ is $\left.1-1\right\}$ via $i$. We define $f: T^{*} \rightarrow \kappa$ by setting $f(t)=i(t)(\alpha)$ for $h t(t, T)=\alpha+1$. Let $s<t \in T^{*}$. Then $h t(s, T)=\beta+1<\alpha+1=h t(t, T)$. Since $i(s) \subset i(t), i(s)(\beta)=i(t)(\beta)$. As $i(t)$ is $1-1, i(t)(\beta) \neq i(t)(\alpha)$. Therefore $f(s) \neq f(t)$.

On the other hand, generalisation of Lemma 2.8 requires the additional assumption that $\kappa^{<\kappa}=\kappa$ since we need to use Generalised Kurepa's Theorem.

Lemma 3.17. Assume $\kappa^{<\kappa}=\kappa$. Let $T$ be a $\kappa^{+}$-Aronszajn tree. $T$ is a normal $\mathbb{R}_{\mathcal{K}^{-}}$ embeddable tree if and only if $T$ is M-special.

Proof. It follows by Theorem 3.13 and Lemmas 3.12 and 3.16.

Unlike special $\omega_{1}$-Aronszajn trees, it is not provable in ZFC that special $\kappa$-Aronszajn trees exist for $\kappa>\omega_{1}$. Hence we are also interested in the question how the existence of one kind of special Aronszajn trees influences the existence of other kinds of special 
Aronszajn trees. The following lemma claims that if there are no M-special Aronszajn trees then there are no special Aronszajn trees at all.

Lemma 3.18. Let $\kappa$ be a regular cardinal. If there exists a special $\kappa^{+}$-Aronszajn tree, then there exists an M-special Aronszajn tree.

Proof. Let $T$ be a special $\kappa^{+}$-Aronszajn tree. We first add one root $r$ such that $r<t$ for each $t \in T_{0}$. Now we wish to guarantee the condition (iv) of Definition 1.5. Let $\alpha<\kappa^{+}$ be a limit ordinal and let $C$ be a cofinal branch in $T \uparrow \alpha$ such that there exists node $t \in T$ greater than all nodes $c \in C$. Then we add one extra node $t_{C}$ to the limit level $\alpha$ such that $t_{C}>c$ for all $c \in C$ and $t_{C}<t$ for all $t>C$, where $t>C$ means $t>c$ for all $c \in C$.

Since for every chain we add one extra node to the limit level, this new tree satisfies (iv). Denote this tree $T^{\prime}$. This tree is normal and $T=\bigcup_{\alpha<\kappa^{+}} T_{\alpha+1}^{\prime}$. By Lemma 3.16 the tree $T^{\prime}$ is $\mathrm{M}$-special.

As in previous section it makes sense to introduce the concept of $\mathcal{S}$-special Aronszajn trees.

Definition 3.19. Let $\kappa$ be a regular cardinal and $S$ be an unbounded subset of $\kappa^{+}$. We say that the $\kappa^{+}$-tree $T$ is $S$-special if $T \uparrow S$ is special, where $T \uparrow S=\{t \in T \mid h t(t, T) \in S\}$ with the induced ordering. We say that a $\kappa^{+}$-tree $T$ is $\mathcal{S}$-special if there is $S$, an unbounded subset of $\kappa^{+}$, such that $T$ is $S$-special. We denote the class of all $\mathcal{S}$-special $\kappa^{+}$-Aronszajn trees as $\mathbb{A}^{\mathcal{S} \text {-sp }}\left(\kappa^{+}\right)$.

The proofs of the following lemmas are direct generalisations of proofs of Facts 2.10 and 2.11 .

Lemma 3.20. Let $C$ be a closed unbounded subset of $\kappa^{+}$, where $\kappa$ is a regular cardinal. If $T$ is a $C$-special $\kappa^{+}$-Aronszajn tree, then $T$ is special.

Proof. Let $T$ be a $C$-special $\kappa^{+}$-Aronszajn tree. Then $T \uparrow C=\bigcup_{v<\kappa} A_{v}$, where each $A_{v}$ is an antichain. Let $\left\{a_{\alpha}^{v} \mid \alpha<\kappa^{+}\right\}$be an enumeration of $A_{v}$ for each $v<\kappa$. Let $\left\{c_{\alpha} \mid \alpha<\kappa^{+}\right\}$ be the monotone enumeration of $C$. For $\alpha<\kappa^{+}$and for $x \in T_{c_{\alpha}}$, we define $S_{x}=\{y \in T \uparrow$ $\left.c_{\alpha+1} \mid x<_{T} y\right\}$. Since each $S_{x}$ has size less than $\kappa^{+}$, let $\left\{s_{\mu}(x) \mid \mu<\kappa\right\}$ be an enumeration of $S_{x}$. Set

$$
A_{v, \mu}=\left\{s_{\mu}\left(a_{\alpha}^{v}\right) \mid \alpha<\kappa^{+}\right\} .
$$

Clearly, $A_{v, \mu}$ is an antichain of $T$ for each $v, \mu<\kappa$. Since $C$ is closed unbounded, $T=\bigcup_{v<\kappa} A_{v} \cup \bigcup_{v, \mu<\kappa} A_{v, \mu}$. Hence $T$ is special.

Lemma 3.21. Let $\kappa$ be a regular cardinal and $S$ be an unbounded subset of $\kappa^{+}$. If every $\kappa^{+}$-Aronszajn tree is $S$-special then every $\kappa^{+}$-Aronszajn tree is special.

Proof. Let $S=\left\{\alpha_{\mu} \mid \mu<\kappa^{+}\right\}$be an unbounded subset of $\kappa^{+}$and $T$ be a $S$-special $\kappa^{+}$ Aronszajn tree. We define a new tree

$$
T^{\prime}=\left\{\langle t, \beta\rangle \mid t \in T \text { and } \beta<\alpha_{h t(t, T)} \text { and } \forall s<t\left(\alpha_{h t(s, T)}<\beta\right)\right\} .
$$

The tree $T^{\prime}$ is ordered by $<_{T^{\prime}}$ as follows: $\langle t, \beta\rangle<_{T^{\prime}}\langle s, \gamma\rangle$ if and only if $t<s$ or $(t=s$ and $\beta<\gamma$ ). It is obvious that $T$ satisfies our definition of Aronszajn tree. Hence $T^{\prime}$ is 
$S$-special, i.e. $T^{\prime} \uparrow S$ is special. Since $T$ is isomorphic to $T^{\prime} \uparrow S=\left\{\left\langle t, \alpha_{h t(t, T)}\right\rangle \mid t \in T\right\}, T$ is special.

Again, note that $\mathcal{S}$-special $\kappa^{+}$-Aronszajn trees are not Suslin in a strong sense. This means that every subset of size $\kappa^{+}$of such tree contains an antichain of size $\kappa^{+}$. Hence we can generalize Definition 2.12 and Lemma 2.14 .

Definition 3.22. Let $\kappa$ be a regular cardinal and $T$ be a $\kappa^{+}$-Aronszajn tree. We say that $T$ is non-Suslin if every subset $U$ of $T$, which has size $\kappa^{+}$, contains an antichain of size $\kappa^{+}$. We denote the class of all non-Suslin Aronszajn trees at $\kappa^{+}$as $\mathbb{A}^{N S}\left(\kappa^{+}\right)$.

The proof of following lemma is a direct generalisation of proof of Fact 2.13.

Lemma 3.23. Let $\kappa$ be a regular cardinal and $T$ be $a \kappa^{+}$-Aronszajn tree. If $T$ is not non-Suslin, then $T$ has a subtree which is Suslin.

Proof. Let $T$ be a $\kappa^{+}$-Aronszajn tree, which is not non-Suslin. Then there is a subset $X$ of $T$ such that $|X|=\kappa^{+}$and $X$ does not contain antichain of size $\kappa^{+}$. We define $T^{\prime}=$ $\{s \in T \mid \exists t \in X(s<t)\}$. It is easy to verify that $T^{\prime}$ is Suslin.

The proof of following lemma is a direct generalisation of proof of Lemma 2.14.

Lemma 3.24. Let $\kappa$ be a regular cardinal and $T$ be a $\kappa^{+}$-Aronszajn tree. If $T$ is $\mathcal{S}$-special, then $T$ is non-Suslin.

The following theorem is only the summary of what we have showed about the relative existence of different kinds of special Aronszajn trees. It tells us that the weak tree property $^{7}$ at $\kappa^{+}$is equivalent to the claim that there are no M-special $\kappa^{+}$-Aronszajn trees and also to the claim that there are no $\mathcal{S}$-special $\kappa^{+}$-Aronszajn trees.

Theorem 3.25. Let $\kappa$ be a regular. The following are equivalent

(i) $\mathbb{A}^{s p}\left(\kappa^{+}\right)=\varnothing$;

(ii) $\mathbb{A}^{M}\left(\kappa^{+}\right)=\varnothing$;

(iii) $\mathbb{A}^{\mathcal{S}-s p}\left(\kappa^{+}\right)=\varnothing$.

Proof. Ad (i) $\Leftrightarrow$ (ii). The claim from left to right follows from Lemma 3.16 and the converse follows from Lemma 3.18.

Ad (i) $\Leftrightarrow$ (iii). This follows from the definition of $\mathcal{S}$-special $\kappa^{+}$-Aronszajn tree.

To sum up:

$$
\mathbb{A}^{s p}\left(\kappa^{+}\right) \subseteq \mathbb{A}^{\mathcal{S}-s p}\left(\kappa^{+}\right) \subseteq \mathbb{A}^{N S}\left(\kappa^{+}\right) \text {and } \mathbb{A}^{M}\left(\kappa^{+}\right) \subseteq \mathbb{A}^{\mathcal{S}-s p}\left(\kappa^{+}\right)
$$

If moreover we only consider normal trees and assume that $\kappa^{<\kappa}=\kappa$, we get:

$$
\mathbb{A}^{s p}\left(\kappa^{+}\right)=\mathbb{T}\left(\mathbb{Q}_{\kappa}\right)\left(\kappa^{+}\right) \subseteq \mathbb{T}\left(\mathbb{R}_{\kappa}\right)\left(\kappa^{+}\right)=\mathbb{A}^{\mathrm{M}-s p}\left(\kappa^{+}\right) \subseteq \mathbb{A}^{\mathcal{S}-s p}\left(\kappa^{+}\right) \subseteq \mathbb{A}^{N S}\left(\kappa^{+}\right)
$$

7 We say that a cardinal $\kappa^{+}$has the weak tree property, if there are no special $\kappa^{+}$-Aronszajn trees. 


\subsection{Existence}

We are interested in special Aronszajn trees at successors of regular cardinals. While the existence of a special $\omega_{1}$-Aronszajn tree can be proved in ZFC, at higher cardinals we need some additional assumption, for example $\kappa^{<\kappa}=\kappa$ or weak square principle. The first one was used in construction by Specker in [Spe49] and the second one in the construction by Jensen in [Jen72]. On the other hand, it is possible to find a model with no special $\kappa^{+}$-Aronszajn tree where $\kappa>\omega$ is regular, but this requires much stronger assumption. Throughout this section we assume that $\kappa$ is a regular cardinal and $\kappa>\omega$.

Definition 3.26. $E_{\kappa}^{\kappa^{+}}=\left\{\alpha<\kappa^{+} \mid c f(\alpha)=\kappa\right\}$

This theorem is our generalisation of Fact 2.15. As a corollary we obtain that the first inclusion in (3.10) can be consistently proper.

Theorem 3.27. Assume $\kappa^{<\kappa}=\kappa$ and $\diamond_{\kappa^{+}}\left(E_{\kappa}^{\kappa^{+}}\right)$. Then there is an $\mathrm{M}$-special $\kappa^{+}$-Aronszajn tree, which is not special.

Proof. By $\diamond_{\kappa^{+}}\left(E_{\kappa}^{\kappa^{+}}\right)$there is a sequence $\left\langle f_{\alpha} \mid \alpha \in E_{\kappa}^{\kappa^{+}}\right\rangle$such that $f_{\alpha}$ is a function from $\alpha$ to $\alpha$ and for any function $f: \kappa^{+} \rightarrow \kappa^{+}$the set $\left\{\alpha \in E_{\mathcal{\kappa}}^{\kappa^{+}} \mid f_{\alpha}=f \uparrow \alpha\right\}$ is stationary in $\kappa^{+}$. We fix this sequence for the rest of the proof.

We construct the tree $T$ and the function $\pi: T \rightarrow \kappa^{+}$, which will code the tree in $\kappa^{+}$, by induction on $\alpha<\kappa^{+}$. For each $\alpha<\kappa^{+}$we require the following conditions:

(T1) If $s \in T \uparrow \alpha$ then $|\kappa \backslash \operatorname{Rng}(s)|=\kappa$.

(T2) If $s \in T \uparrow \alpha$ and $x \in[\kappa \backslash \operatorname{Rng}(s)]^{<\kappa}$ then there is $s^{\prime} \supseteq s$ on each higher level of $T \uparrow \alpha$ such that $\operatorname{Rng}\left(s^{\prime}\right) \cap x=\varnothing$.

$(\pi 0) \pi_{\alpha}$ is a 1-1 map from $T \uparrow \alpha$ to $\kappa^{+}$such that $s \subseteq t \rightarrow \pi_{\alpha}(s)<\pi_{\alpha}(t)$ and for $\beta<\alpha$, $\pi_{\beta} \subseteq \pi_{\alpha}$.

Let $T_{0}=\{\varnothing\}$ and $\pi_{1}$ is an arbitrary function from $T \uparrow 1=T_{0}$ to $\kappa^{+}$. It is clear that $T_{0}$ satisfies both conditions and $\pi_{1}$ satisfies $(\pi 0)$.

Let $\alpha=\beta+1$. Suppose $T \uparrow(\beta+1)$ and $\pi_{\beta+1}$ are defined and they satisfy the conditions mentioned above. We want to construct level $T_{\alpha}$. For each $s \in T_{\beta}$ we add all one-point extensions $s \cup\{\langle\alpha, v\rangle\}$ of $s$ such that $v \in \kappa \backslash \operatorname{Rng}(s)$. This is possible by (T1), which guarantees the existence of $\kappa$-many such extensions. Since we add all such extension of $s$, for each $x \in[\kappa \backslash \operatorname{Rng}(s)]^{<\kappa}$ we can always find $t \in T_{\alpha}$ such that $s \subseteq t$ and $x \cap \operatorname{Rng}(s)=\varnothing$; therefore $T \uparrow(\alpha+1)$ satisfies (T2). As $T \uparrow(\beta+1)$ satisfies (T1), $T \uparrow(\alpha+1)$ satisfies (T1), too. To obtain $\pi_{\alpha+1}$, we extend $\pi_{\alpha}$ arbitrarily such that it satisfies the condition $(\pi 0)$.

Let $\alpha$ be limit. For each $\beta<\alpha$, suppose $T \uparrow \beta$ and $\pi_{\beta}$ are defined and they satisfy the conditions mentioned above. We need to distinguish two cases. First, if $\alpha$ has cofinality less than $\kappa$ then we add all possible sequences. We can do that since $\kappa^{<\kappa}=\kappa$.

Second, if $\alpha$ has cofinality $\kappa$ then let $T_{\alpha}^{\prime}=\bigcup_{\beta<\alpha} T_{\beta}$ and $\pi_{\alpha}^{*}=\bigcup_{\beta<\alpha} \pi_{\beta}$. We construct for each $s \in T_{\alpha}^{\prime}$ and $x \in[\kappa \backslash \operatorname{Rng}(s)]^{<\kappa}$ node $s_{x}$ above $s$ of height $\alpha$ such that $x \cap \operatorname{Rng}(s)=\varnothing$. Let us fix for the rest of the proof a bijection $g$ from $\kappa$ to $\mathbb{Q}_{\kappa}$. Again, we need to distinguish two cases. First, if $f_{\alpha} \circ g$ embeds $\pi_{\alpha}^{* \prime \prime} T_{\alpha}^{\prime}$ to $\mathbb{Q}_{\kappa}$ and $\operatorname{Dom}\left(f_{\alpha}\right)=\pi_{\alpha}^{* \prime \prime} T_{\alpha}^{\prime}$, then set

$$
X_{\alpha}=\left\{(s, x) \mid s \in T_{\alpha}^{\prime} \& x \in[\kappa]^{<\kappa} \& \operatorname{Rng}(s) \cap x=\varnothing\right\} .
$$


For $(s, x),(t, y)$ in $X_{\alpha}$, we define $(s, x) \leq_{\alpha}(t, y)$ if and only if $s \subseteq t$ and $x \subseteq y$. For each $q \in \mathbb{Q}_{\kappa}$, set

$$
\begin{aligned}
\Delta_{q}^{\alpha}=\left\{(s, x) \in X_{\alpha} \mid g\left(f_{\alpha}\left(\pi_{\alpha}^{*}(s)\right)\right) \geq_{\mathbb{Q}} q\right. \text { or } & \\
& \left.\left(\forall(t, y) \in X_{\alpha}\right)\left((t, y) \geq_{\alpha}(s, x) \rightarrow g\left(f_{\alpha}\left(\pi_{\alpha}^{*}(t)\right)\right)<_{\mathbb{Q}_{\kappa}} q\right)\right\} .
\end{aligned}
$$

It is easy to see that $\Delta_{q}^{\alpha}$ is cofinal in $X_{\alpha}$.

Let $s \in T_{\alpha}^{\prime}$ and $x \in[\kappa \backslash \operatorname{Rng}(s)]^{<\kappa}$ be given. First we fix an increasing sequence $\left\langle\alpha_{\gamma}\right| \gamma\langle\kappa\rangle$ with limit $\alpha$ and $\alpha_{0}=$ length $(s)$. By induction we construct an increasing sequence $\left\langle\left(s_{\gamma}, x_{\gamma}\right) \Delta_{g(\gamma)}^{\alpha} \mid \beta<\kappa\right\rangle$ with length $\left(s_{\gamma}\right) \geq \alpha_{\gamma}$ for all $\gamma<\kappa$.

Let $s_{0}^{\prime}=s$ and $x_{0}^{\prime}=x$. By definition of $X_{\alpha},\left(s_{0}^{\prime}, x_{0}^{\prime}\right)$ is in $X_{\alpha}$ and as $\Delta_{g(0)}^{\alpha}$ is cofinal in $X_{\alpha}$, we can find $\left(s_{0}, x_{0}\right) \geq_{\alpha}\left(s_{0}^{\prime}, x_{0}^{\prime}\right)$ in $\Delta_{g(0)}^{\alpha}$.

If $\gamma<\kappa$ is a successor ordinal $\gamma=\beta+1$ we can proceed as follows. Assume $\left(s_{\beta}, x_{\beta}\right)$ is defined. By (T1) there is $v_{\beta} \in \kappa \backslash\left(\operatorname{Rng}\left(s_{\beta}\right) \cup x_{\beta}\right)$. Let $x_{\beta+1}^{\prime}=x_{\beta} \cup\left\{v_{\beta}\right\}$. By (T2) we can find $s_{\beta+1}^{\prime} \in T_{\alpha}^{\prime}$ such that $s_{\beta+1}^{\prime} \supseteq s_{\beta}$, length $\left(s_{\beta+1}^{\prime}\right) \geq \alpha_{\beta+1}$ and $\operatorname{Rng}\left(s_{\beta+1}^{\prime}\right) \cap x_{\beta}^{\prime}=\varnothing$. By definition of $X_{\alpha},\left(s_{\beta}^{\prime}, x_{\beta}^{\prime}\right)$ is in $X_{\alpha}$ and as $\Delta_{g(\beta)}^{\alpha}$ is cofinal in $X_{\alpha}$, we can find $\left(s_{\beta+1}, x_{\beta+1}\right) \geq_{\alpha}\left(s_{\beta+1}^{\prime}, x_{\beta+1}^{\prime}\right)$ in $\Delta_{g(\beta+1)}^{\alpha}$.

Let $\gamma<\kappa$ be limit. Since $\gamma<\kappa$ we can take $s_{\gamma}^{\prime \prime}=\bigcup_{\beta<\gamma} s_{\beta}$ and $x_{\gamma}^{\prime}=\bigcup_{\beta<\gamma} x_{\beta}$. As $\kappa$ is regular, $\left|x_{\gamma}^{\prime}\right|<\kappa$. Note that $\operatorname{Rng}\left(s_{\gamma}^{\prime \prime}\right) \cap x_{\gamma}^{\prime}=\varnothing$, but length $\left(s_{\gamma}^{\prime \prime}\right)$ does not have to be greater or equal to $\alpha_{\gamma}$. However, by (T2) there exists $s_{\gamma}^{\prime} \supseteq s_{\gamma}^{\prime \prime}$ such that $\operatorname{Rng}\left(s_{\gamma}^{\prime}\right) \cap x_{\gamma}^{\prime}=\varnothing$ and length $\left(s_{\gamma}^{\prime}\right) \geq \alpha_{\gamma}$. By definition of $X_{\alpha},\left(s_{\gamma}^{\prime}, x_{\gamma}^{\prime}\right)$ is in $X_{\alpha}$ and as $\Delta_{g(\gamma)}^{\alpha}$ is cofinal in $X_{\alpha}$, we can find $\left(s_{\gamma}, x_{\gamma}\right) \geq_{\alpha}\left(s_{\gamma}^{\prime}, x_{\gamma}^{\prime}\right)$ in $\Delta_{g(\gamma)}^{\alpha}$.

In the other case, if $f_{\alpha} \circ g$ does not embed $\pi_{\alpha}^{* \prime \prime} T_{\alpha}^{\prime}$ to $\mathbb{Q}_{\kappa}$, then we proceed similar as before. Let $s \in T_{\alpha}^{\prime}, x \in[\kappa \backslash \operatorname{Rng}(s)]^{<\kappa}$ and $\left\langle\alpha_{\gamma} \mid \gamma<\kappa\right\rangle$ be cofinal in $\alpha$ with $\alpha_{0}=$ length $(s)$. By induction we construct an increasing sequence $\left\langle\left(s_{\gamma}, x_{\gamma}\right) \mid \beta<\kappa\right\rangle$ with length $\left(s_{\gamma}\right) \geq \alpha_{\gamma}$ for all $\gamma<\kappa$.

Let $s_{0}=s$ and $x_{0}=x$.

If $\gamma<\kappa$ is a successor ordinal $\gamma=\beta+1$ we can proceed as follows. Assume $\left(s_{\beta}, x_{\beta}\right)$ is defined. By (T1) there is $v_{\beta} \in \kappa \backslash\left(\operatorname{Rng}(s) \cup x_{\beta}\right)$. Let $x_{\beta+1}=x_{\beta} \cup\left\{v_{\beta}\right\}$. By (T2) we can find $s_{\beta+1} \in T_{\alpha}^{\prime}$ such that $s_{\beta+1} \supseteq s_{\beta}$, length $\left(s_{\beta+1}\right) \geq \alpha_{\beta+1}$ and $\operatorname{Rng}\left(s_{\beta+1}\right) \cap x_{\beta+1}=\varnothing$.

Let $\gamma<\kappa$ be limit. Since the size of $\gamma$ is less than $\kappa$, we can take $s_{\gamma}^{\prime}=\bigcup_{\beta<\gamma} s_{\beta}$ and $x_{\gamma}=\bigcup_{\beta<\gamma} x_{\beta}$. As $\kappa$ is regular, $\left|x_{\gamma}\right|<\kappa$. Note that $\operatorname{Rng}\left(s_{\gamma}^{\prime}\right) \cap x_{\gamma}=\varnothing$, but length $\left(s_{\gamma}^{\prime}\right)$ does not have to be greater or equal to $\alpha_{\gamma}$. However by (T2) there exist $s_{\gamma} \supseteq s_{\gamma}^{\prime}$ such that $\operatorname{Rng}\left(s_{\gamma}\right) \cap x_{\gamma}=\varnothing$ and length $\left(s_{\gamma}\right) \geq \alpha_{\gamma}$.

Let $s_{x}=\bigcup_{\gamma<\kappa} s_{\gamma}$. We define the level $T_{\alpha}=\left\{s_{x} \mid s \in T_{\alpha}^{\prime}\right.$ and $\left.x \in[\kappa \backslash \operatorname{Rng}(s)]^{<\kappa}\right\}$. It is easy to verify that $T \uparrow(\alpha+1)=T_{\alpha}^{\prime} \cup T_{\alpha}$ satisfies the condition (T1) and (T2). Again, we can extend $\pi_{\alpha}^{*}$ to $\pi_{\alpha+1}$ on $T \uparrow(\alpha+1)$ arbitrarily such that it satisfies the condition $(\pi 0)$.

Finally, set $T=\bigcup_{\alpha<\kappa^{+}} T_{\alpha}$ and $\pi=\bigcup_{\alpha<\kappa^{+}} \pi_{\alpha}$. Then $\pi: T \rightarrow \kappa^{+}$is a function such that $s \subseteq t \rightarrow \pi(s)<\pi(t)$. 
For a contradiction assume that $T$ is special. As we assume $\kappa^{<\kappa}=\kappa$, by Lemma 3.12 $T$ is special if and only if $T$ is $\mathbb{Q}_{\kappa}$-embeddable. Therefore there is a function $f: \kappa^{+} \rightarrow \kappa$ such that $f \circ g$ embeds $\pi^{\prime \prime} T$ in $\mathbb{Q}$. Let

$$
C=\left\{\alpha<\kappa^{+} \mid \alpha \text { is a limit ordinal and } \pi^{\prime \prime}(T \uparrow \alpha)=\pi_{\alpha}^{* \prime \prime} T_{\alpha}^{\prime}\right. \text { and }
$$

$$
\begin{aligned}
& f \circ g\left\lceil\alpha \text { embeds } \pi_{\alpha}^{* \prime \prime} T_{\alpha}^{\prime} \text { in } \mathbb{Q}_{\kappa}\right. \text { and } \\
& \left(\forall s \in T_{\alpha}^{\prime}\right)\left(\forall x \in[\kappa \backslash \operatorname{Rng}(s)]^{<\kappa}\right)\left(\forall q>_{\mathbb{Q}} g(f(\pi(s)))\right. \\
& \left((\exists t \in T)\left(t \supseteq s \& \operatorname{Rng}(t) \cap x=\varnothing \& g(f(\pi(t))) \geq_{\mathbb{Q}} q\right)\right. \\
& \left.\rightarrow\left(\exists t^{\prime} \in T_{\alpha}^{\prime}\right)\left(t^{\prime} \supseteq s \& \operatorname{Rng}\left(t^{\prime}\right) \cap x=\varnothing \& g\left(f\left(\pi\left(t^{\prime}\right)\right)\right) \geq_{\mathbb{Q}} q\right)\right\} .
\end{aligned}
$$

It is easy to verify that $C$ is a closed unbounded subset of $\kappa^{+}$. As we assume $\nabla_{\kappa}\left(E_{\kappa}^{\kappa^{+}}\right)$, the set $\left\{\alpha \in E_{\mathcal{K}}^{\kappa^{+}} \mid f \uparrow \alpha=f_{\alpha}\right\}$ is stationary, so there is $\alpha \in C$ such that $f \uparrow \alpha=f_{\alpha}$ and $\alpha$ has cofinality $\kappa$. Let $t \in T_{\alpha}$ and let $q=g(f(\pi(t)))$. By the construction of $T$, there is $(s, x) \in \Delta_{q}^{\alpha}$ such that $\operatorname{Rng}(s) \cap x=\varnothing$ and $s \subset t$. Since $f \circ g$, and $\pi$ are order-preserving, $g(f(\pi(s)))<_{\mathbb{Q}} g(f(\pi(t)))=q$.

Since $g(f(\pi(s)))<_{\mathbb{Q}} q$ and $g(f(\pi(t))) \geq_{\mathbb{Q}} q$, by the definition of $C$ there exists $t^{\prime} \in T_{\alpha}^{\prime}$ such that $t^{\prime} \supseteq s, \operatorname{Rng}\left(t^{\prime}\right) \cap x=\varnothing$ and $g\left(f\left(\pi\left(t^{\prime}\right)\right)\right) \geq_{\mathbb{Q}} q$. Note that $(s, x),\left(t^{\prime}, x\right)$ are in $X_{\alpha}$ and $(s, x) \leq_{\alpha}\left(t^{\prime}, x\right)$. Since $(s, x)$ is in $\Delta_{q}^{\alpha}$ and $f \uparrow \alpha=f_{\alpha}$, by (3.13) it must hold that $g\left(f_{\alpha}(\pi(s))\right) \geq_{\mathbb{Q}} q$. But $f_{\alpha}=f \uparrow \alpha$ and so $g(f(\pi(s))) \geq_{\mathbb{Q}} q$. This contradicts our earlier inequality $g(f(\pi(s)))<_{\mathbb{Q}} q$.

Corollary 3.28. Assume $\kappa^{<\kappa}=\kappa$ and $\diamond_{\kappa^{+}}\left(E_{\kappa}^{\kappa^{+}}\right)$. Then there is an $\mathbb{R}_{\kappa}$-embeddable $\kappa^{+}$-Aronszajn tree, which is not special.

Proof. By Lemma 3.17, every M-special $\kappa^{+}$-Aronszajn tree is $\mathbb{R}_{\kappa}$-embeddable.

Corollary 3.29. Assume $\kappa^{<\kappa}=\kappa$ and $\mho_{\mathcal{K}^{+}}\left(E_{\mathcal{\kappa}}^{\kappa^{+}}\right)$. Then there is an $\mathcal{S}$-special $\kappa^{+}$-Aronszajn tree, which is not special.

Proof. By Lemma 3.16, every M-special $\kappa^{+}$-Aronszajn tree is $\mathcal{S}$-special for $S=\{\alpha+$ $\left.1 \mid \alpha<\kappa^{+}\right\}$.

The next lemma is a straightforward generalisation of Lemma 2.16 and tells us that the last inclusion in (3.10) can be consistently proper.

Lemma 3.30. Assume $\kappa^{<\kappa}=\kappa$ and $\diamond_{\kappa^{+}}\left(E_{\kappa}^{\kappa^{+}}\right)$. Then there is a $\kappa^{+}$-Aronszajn tree, which is $S$-special for some $S$ unbounded subset of $\kappa^{+}$and it is not M-special and by our assumption it is not $\mathbb{R}_{\kappa}$-embeddable.

Proof. The proof is the same as in Lemma 2.16.

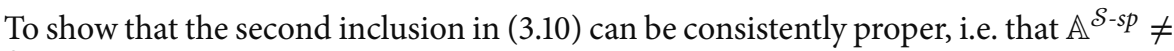
$\mathbb{A}^{N S}$, we need to introduce the notion of an $\omega$-ascent path, which is due to Laver.

Definition 3.31. Let $\kappa$ be a regular cardinal. We say that a $\kappa^{+}$-Aronszajn tree $T$ has the property of the $\omega$-ascent path if there is a sequence $\left\langle x^{\alpha} \mid \alpha<\kappa^{+}\right\rangle$such that

(i) for each $\alpha<\kappa^{+}, x^{\alpha}$ is a function from $\omega$ to $T_{\alpha}$;

(ii) if $\alpha, \beta<\kappa$ with $\alpha<\beta$ then $\exists n \in \omega \forall m \geq n x_{m}^{\alpha}<x_{m}^{\beta}$. 
If the tree $T$ has a cofinal branch, then this branch is a 1-ascent path and it is obvious that $T$ is not special. But Aronszajn trees do not have cofinal branches. Thus an $\omega$-ascent path is a pseudo-branch with width $\omega$ which prevents the tree from being special.

The following fact is due to Shelah ([SS88]), building on work of Laver and Todorčević.

Fact 3.32. Let $\kappa>\omega$ be a regular cardinal. Let $T$ be a $\kappa^{+}$-Aronszajn tree with the property of an $\omega$-ascent path. Then $T$ is not special.

Remark 3.33. No such argument can exist for $\omega_{1}$-trees since it is important for the proof that there is a regular cardinal between $\omega$ and $\kappa^{+}$. This is the difference between the specialization forcing for $\omega_{1}$ and for higher cardinals. In the case of higher cardinals, if $T$ has an $\omega$-ascent path, then any specialization forcing must collapse cardinals. On the other hand, as was pointed out by a referee, Baumgartner showed that an $\omega_{1}$-tree has a cofinal branch if and only if it contains an ascent path of finite width. In particular, the nonexistence of paths of finite width implies that the corresponding specialization forcing has the ccc.

Corollary 3.34. Let $\kappa$ be a regular cardinal. Let $T$ be a $\kappa^{+}$-Aronszajn tree with the property of an $\omega$-ascent path. Then $T$ is not $\mathcal{S}$-special.

Proof. Let $S \subseteq \kappa^{+}$be an unbounded subset of $\kappa^{+}$and $\left\langle x^{\alpha} \mid \alpha<\kappa^{+}\right\rangle$be an $\omega$-ascent path. Then $\left\langle x^{\alpha} \mid \alpha<\kappa^{+}\right\rangle \uparrow S$ is $\omega$-ascent path for $T\lceil S$ and by the previous theorem $T\lceil S$ is not special.

The construction of the following tree can be found in [SS88]. ${ }^{8}$

Fact 3.35. Let $\kappa$ be a regular cardinal. Assume $\square_{\kappa}$. Then there is a non-Suslin $\kappa^{+}-$ Aronszajn tree with $\omega$-ascent path.

Hence we can conclude that the second inclusion in (3.10) can be consistently proper.

Corollary 3.36. Let $\kappa$ be a regular cardinal. Assume $\square_{\kappa}$. Then there is a non-Suslin $\kappa^{+}$-Aronszajn tree $T$ such that $T$ is not $\mathcal{S}$-special.

Proof. It follows from Corollary 3.34 and Fact 3.35.

\section{References}

[Bau70] James Baumgartner. Decomposition and embeddings of trees. Notices of the American Mathematical Society, 17, 1970.

[BMR70] James Baumgartner, Jerome Malitz, and William Reinhardt. Embedding Trees in the Rationals. Proceedings of the National Academy of Sciences, 67(4): 1748-1753, 1970.

[Dev72] Keith Devlin. Note on a theorem of J. Baumgartner. Fundamenta Mathematicae, 76(3): 255-260, 1972.

[DJ74] Keith Devlin and Håvard Johnsbråten. The Souslin Problem. Lecture Notes in Mathematics. SpringerVerlag, Berlin, third edition, 1974. The third millennium edition, revised and expanded.

[Han81] Masazumi Hanazawa. Various Kinds of Aronszajn Tree with No Subtree of a Different Kind. Lecture Notes in Mathematics, 891: 1-21, 1981.

8 It is worth noting that Todorčević also constructed Aronszajn trees with ascent paths from weaker assumptions, see [Tod89]. 
[Jec03] Thomas Jech. Set Theory. Springer Monographs in Mathematics. Springer-Verlag, Berlin, 2003.

[Jen72] Ronald Jensen. The Fine Structure of The Constructible Hierarchy. Annals of Mathematical Logic, 4: 229-308, 1972.

[Kur35] Djuro R. Kurepa. Ensembles ordonnés et ramifiés. Publications mathématiques de l'Université de Belgrade, 4: 1-138, 1935.

[Mit72] William Mitchell. Aronszajn Trees and Independence of the Transfer Property. Annals of Mathematical Logic, 5: 21-46, 1972.

[Sch14] Chaz Schlindwein. How Special is your Aronszajn Tree? http://arxiv.org/pdf/math/0312445vl.pdf, 2014.

[She98] Saharon Shelah. Proper and Improper Forcing. Perspectives in Mathematical Logic. Springer-Verlag, Berlin, second edition, 1998.

[Spe49] Ernst Specker. Sur un problème de Sikorski. Colloquium Mathematicum, 2: 9-12, 1949.

[SS88] Saharon Shelah and Lee Stanley. Weakly Compact Cardinals and Nonspecial Aronszajn Trees. Proceedings of the American Mathematical Society, 104(3): 887-897, 1988.

[Tod84] Stevo Todorčević. Trees and linearly ordered sets. In Kenneth Kunen and Jerry Vaughan, editors, Handbook of Set-Theoretic Topology. Elsevier Science Publisher B.V., 1984.

[Tod89] Stevo Todorčević. Special square sequences. Proc. Amer. Math. Soc., 105(1): 199-205, 1989. 